\title{
Wohlfahrt und Altruismus - Eine Bestandsaufnahme aus der experimentellen Wirtschaftsforschung für die Politikwissenschaft*
}

Können Menschen altruistisch handeln? Anhand von Ultimatumspielen oder Public-GoodsSpielen lässt sich zeigen, dass Probanden in Laborexperimenten unter bestimmten Bedingungen freiwillig Kosten auf sich nehmen, um zu kooperieren oder um unfair handelnde Spielpartner zu bestrafen. Dieses Verhalten interpretieren einige Forscher als altruistisches Verhalten, andere Wissenschaftler widersprechen dieser Sichtweise. Durch die Analyse konkurrierender Theorien sowie einiger der sie stützenden experimentellen Daten kann gezeigt werden, dass auch sogenannte ,, altruistische Handlungsweisen “immer auf trainierten Varianten strategischer Interaktion beruhen. Diese Erkenntnis ist für die politikwissenschaftliche Feldforschung sehr bedeutsam. Für den Bereich der Sozialpolitik lässt sich damit z. B. erklären, warum in Zeiten neu aufbrechender Verteilungskonflikte häufig eine Beschneidung sozialpolitischer Maßnahmen unterstützt wird. Eine Orientierung an den Theorien und Methoden der experimentellen Wirtschaftsforschung kann so zu einem tieferen Verständnis des Zusammenhangs zwischen individuellem Verhalten und politischen Makrophänomenen in der Politikwissenschaft führen.

Inhalt

1. Einleitung

2. Experimentelle Wirtschaftsforschung und ihre Relevanz für die Politikwissenschaft 787

a) Wie funktionieren Laborexperimente? 789

b) Einfache Ultimatumspiele, Diktatorspiele und Public-Goods-Spiele $\quad 789$

c) Altruismus in den neueren spieltheoretischen Ansätzen 793

3. Verteilungskonflikte in westeuropäischen Sozialstaaten 797

4. Kostenexplosion und Verteilungsgerechtigkeit 798

5. Fazit $\quad 801$

$\begin{array}{ll}\text { Literatur } & 802\end{array}$

* Für hilfreiche Kommentare und Unterstützung danke ich Maurizio Cotta, Jan Sauermann, Luca Verzichelli, den Mitarbeitern und Doktoranden des Centro Interdipartimentale di Ricerca sul Cambiamento Politico der Universität Siena sowie zwei anonymen Gutachtern. Der Fritz Thyssen Stiftung danke ich für die Finanzierung meines Forschungsaufenthaltes in Siena. Zudem danke ich Ernst Fehr für die Bereitstellung der Daten zu den Public-Goods-Experimenten. 
Ulrich Glassmann

\section{Einleitung}

Sind Menschen zu altruistischen Handlungen fähig? Diese Frage wird erstaunlicherweise immer häufiger in der Wirtschaftswissenschaft gestellt, und was noch erstaunlicher ist: Sie wird dort zum Teil positiv beantwortet (Fehr/Fischbacher 2003; Bowles/Gintis 2006). Während sich in der Sozialwissenschaft zuweilen hartnäckig die Vorstellung hält, normative wie deskriptive ökonomische Theorien gingen immer von dem Axiom der materiellen Eigennutzmaximierung aus, sieht die Realität der wirtschaftswissenschaftlichen Theoriebildung schon seit einiger Zeit ganz anders aus. Die Grundannahme, dass der Mensch sich wie ein homo oeconomicus verhält, wird verschiedentlich bezweifelt. In der experimentellen Forschung finden sich hinreichend empirische Belege dafür, dass Menschen unter bestimmten Voraussetzungen freiwillig auf Geld verzichten (Bolton/Ockenfels 2000).

Ich gehe in diesem Beitrag der Frage nach, inwieweit diese Forschungsergebnisse den Schluss zulassen, menschliches Kooperationsverhalten basiere auf altruistischen Handlungsmotiven. Altruismus wird häufig als eine „unbedingte Freundlichkeit“" gegenüber anderen Menschen aufgefasst, die nicht durch das strategische Interesse eines Akteurs gesteuert wird, sondern eine vorrangige Interessiertheit am Gemeinwohl zum Ausdruck bringt (Fehr/Gächter 2000a). Ich analysiere in diesem Beitrag, ob es in Experimenten Hinweise auf eine solche Verhaltensform gibt.

In der Literatur existiert jedoch auch noch eine hiervon etwas verschiedene Definition des Altruismusbegriffes. Gintis (2003) und einige andere Forscher nennen den Wunsch, auf eigene Kosten zu kooperieren oder zu bestrafen, altruistisch und unterstellen damit zumindest, dass diese Verhaltensform als Ergebnis einer durch soziale Normen forcierten Reziprozität entsteht, also nicht ganz unabhängig von dem Verhalten anderer Akteure auftritt. Auch diese These wird im Folgenden erörtert.

In der Wirtschaftsforschung wird darüber gestritten, ob altruistische Motive von entscheidender Bedeutung sind. Bolton und Ockenfels (2000: 189) halten die Altruismusthese für unzutreffend, weil sie die Bereitschaft zu teilen mit strategischer Fairness erklären können. Dieses Verhalten zielt darauf ab, ungleiche Verhältnisse in strategischer Absicht zu reduzieren. Hingegen nehmen Gintis (2003) und andere Forscher an, dass Umverteilungsprogramme in modernen Gesellschaften sowie die meisten anderen Kooperationsformen zwischen Fremden eher auf ein altruistisches Verhaltensmuster zurückzuführen sind (Fong/Bowles/Gintis 2005).

Diese Altruismustheorie erklärt einerseits die Existenz großzügiger sozialpolitischer Programme, zeigt aber andererseits ebenso auf, weshalb neu aufbrechende Verteilungskonflikte sich immer weiter verschärfen können. Denn Opferbereitschaft ist an eine Erwartung der gerechten Verteilung des Opfers gebunden. Je mehr sich ein Verteilungskonflikt zuspitzt und dadurch spezifische Verlierergruppen erzeugt, desto mehr können altruistische Handlungsmotive eine Verweigerung gegenüber sozialpolitischen Ausgleichsmaßnahmen mobilisieren. Der Altruismusbegriff ist jedoch unter anderem wegen dieser Paradoxie ein sehr umkämpfter und von vielen Forschern abgelehnter Begriff. Daher analysiere ich im Folgenden, welche empirischen Belege die experimentelle Wirtschaftsforschung für die Altruismusannahme liefert und wie sie dabei methodisch vorgeht. Dann stelle ich drei grundlegende Theorien und Modelle dieses Forschungszweiges vor und erläutere mithilfe einer empi- 
rischen Untersuchung neu auftauchender Verteilungskonflikte den Nutzen solcher Theorien für die politikwissenschaftliche Analyse des Sozialstaates.

\section{Experimentelle Wirtschaftsforschung und ihre Relevanz für die Politikwissenschaft}

„Why are empirically minded political scientists so resistant to experimental investigation or, conversely, so taken by observational studies?" (Green/Gerber 2003: 101). Das experimentelle Design verfügt gegenüber Beobachtungs- und Befragungstechniken über den Vorzug, unabhängige Variablen unter kontrollierten Bedingungen systematisch variieren zu können, sodass ihr Effekt verlässlich getestet werden kann. Der „,verdeckte“ Einfluss intervenierender Variablen kann so in hohem Maße ausgeschlossen werden. Bei der Anwendung von Beobachtungs- und Befragungstechniken kann der Forscher dieses Problem nur lösen, indem er Annahmen über den möglichen Einfluss intervenierender Variablen macht. Übersieht er einen wichtigen Zusammenhang, scheitert seine Analyse bereits an der Auswahl der untersuchten Merkmale. Daher bilden Experimente immer noch die avancierteste Methode für kausale Inferenz (Green/Gerber 2003: 99). Diese Methode ist jedoch in der Wirtschaftsforschung sehr viel verbreiteter als in der Politikwissenschaft. Warum?

Zunächst lässt sich zwischen Feld- und Laborexperiment unterscheiden. Die Attraktivität des Feldexperiments (auch social experiment) besteht in seinem direkten realweltlichen Bezug: ,[...] social experiments have one great advantage over laboratory experiments: they are dealing with real-life, and the topics chosen are directly related to issues of policy concern" (Frey/Bohnet 1996: 346). So wurden beispielsweise in den USA häufig Feldexperimente durchgeführt, um Regierungsprogramme zu evaluieren. Dabei ging es darum zu erfahren, ob die Teilnahme an bestimmten Weiterbildungsmaßnahmen tatsächlich einen positiven Arbeitsmarkteffekt für die Beteiligten erbringt oder welchen Effekt Subventionen, Werbung oder das Familieneinkommen auf solche Maßnahmen haben. Durch die Kontrollgruppe, deren Mitglieder nicht an der Maßnahme teilnehmen, produziert das Experiment „,counterfactuals“ zu den Ergebnissen in der Teilnehmergruppe, wobei die Zufallsauswahl der Probanden gewährleistet, dass die Ergebnisse nicht systematisch durch dritte Variablen verzerrt werden. Am Ende eines solchen Experiments lassen sich die Effekte einer Maßnahme relativ einfach als Differenz der erreichten Mittelwerte beider Gruppen abbilden (Heckman/Smith 1996).

Bestünde die experimentelle Wirtschaftsforschung vorwiegend aus solchen Feldexperimenten, dann hätte sich vielleicht eine größere wechselseitige Interessiertheit unter den Politikwissenschaftlern und den Wirtschaftsforschern an den Untersuchungen der anderen Disziplin eingestellt. Die Entwicklung ist aber genau anders herum verlaufen: „,Experiments“ today are understood in a narrow sense: they include laboratory work only and leave social experiments aside [...] The literature on the two types of experiments is neatly separated, or in other words, the two approaches are essentially isolated from each other" (Frey/Bohnet 1996: 346).

Natürlich existieren Ausnahmen. So hat zum Beispiel Armin Falk in einem Feldexperiment nachgewiesen, dass Personen, die von einer karitativen Einrichtung um eine Spende gebeten werden, im Aggregat betrachtet häufiger und insgesamt mehr spenden, wenn sie zu der Spendenaufforderung ein Geschenk erhalten und noch mehr, wenn sie statt eines kleineren 
Geschenks ein größeres Geschenk erhalten (Falk 2004). Solche Einrichtungen sind in der Praxis also gut beraten, wenn sie ihrer Spendenaufforderung ein Geschenk beilegen.

Die Studie von Falk bestätigt zudem die Laborergebnisse von gift-exchange games, in denen Käufer durch die Zahlung eines hohen Preises eine über den Vertrag hinausgehende Gegenleistung erhalten, und bildet eine Brücke zwischen Labor und Feld. Jedoch wird diese Brücke von Politikwissenschaftlern äußerst selten betreten, was unter anderem an der immer noch vorhandenen Skepsis unter manchen Politologen gegenüber formalen Theorien und formaler Modellierung liegt (Fiorina/Plott 1978).

Formale Modelle können dazu dienen, menschliches Verhalten in der Form von Strategiegleichgewichten vorherzusagen. Theorien in diesem Bereich sind also in hohem Maße an eine mathematische Beweisführung für die denkbare Etablierung eines solchen Gleichgewichtes geknüpft. Laborexperimente liefern für die Überprüfung solcher Theorien in idealer Weise Daten. Die allgemeine Kritik hieran lautet, dass formale Modelle eine reine Gedankenwelt abbilden (Schram 2005), deren reduzierte Vorstellungen über die Realität keine Erklärungen für realweltliche Phänomene liefern. „In so far as the research topics of experimental economics are theory-driven, ,relevance" is no prevalent criterion nor is it required that the experimental setting is related to real-world problems" (Frey/Bohnet 1996: 344).

Ich teile diese Kritik nicht, weil nur dadurch, dass die experimentelle Analyse realweltliche Bedingungen zunächst ausschaltet, zuverlässige kausale Schlüsse über menschliches Verhalten möglich sind. Wie diese Verhaltensmuster in historisch kontingenten Zusammenhängen variieren, ist nicht die vorangehende Frage, sondern eine Folgefrage. Theorien, die bereits im Labor versagen, haben keine gute Aussicht, die Feldforschung zu bereichern. Die experimentelle Analyse bietet also in ganz verschiedenen sozialwissenschaftlichen Gebieten einige Möglichkeiten, die Erklärungskraft theoretischer Ansätze zu überprüfen.

Dieser Annahme folgend haben in den siebziger Jahren amerikanische Politologen am California Institute of Technology damit begonnen, Ausschussentscheidungen experimentell $\mathrm{zu}$ untersuchen (Fiorina/Plott 1978). Sie fanden heraus, dass das räumliche Konzept des Kerns (Core) Ausschussentscheidungen mit Mehrheitsregel am besten vorhersagt. Als Kern wird diejenige Menge von Auszahlungsprofilen bezeichnet, die von keiner anderen Menge geschlagen werden kann. Wie Eavey und Miller (1984) später zeigten, lag die Vorhersagekraft des Kerns auch an seinen Fairnesseigenschaften. Wenn der Kern gleichzeitig eine Menge ist, die einen ,guten“ Kompromiss erlaubt, weil darin alle Entscheidungsträger eine faire Auszahlung realisieren können, dann lässt sich das Abstimmungsverhalten mit dem Konzept des Kerns gut modellieren. Verfügt der Kern jedoch nicht über diese Fairnesseigenschaft, dann schwindet auch seine Vorhersagekraft. Die Probanden suchten am häufigsten den fairen Kompromiss, auch wenn das Auszahlungsprofil der Entscheidung nicht im Kern lag (Palfrey 2006).

Damit deutete sich in der experimentell geleiteten Politikwissenschaft die Diskussion über eine Verhaltensweise an, die einige Wissenschaftler später als Altruismus bezeichneten. Die vorwiegend amerikanisch geprägte experimentelle Politikwissenschaft wandte sich in den achtziger Jahren jedoch zunächst spezifischen Fragen des Wählerverhaltens, der Informationsverarbeitung von Akteuren sowie Problemen der politischen Partizipation zu (Palfrey 2006: 921). Erst in jüngerer Zeit wagen sich experimentelle Forscher immer häufiger 
an die Erklärung von Makrophänomenen. Im Rahmen dieser neuen Analysen hat sich die Frage ergeben, ob es zwischen fairem oder altruistischem Verhalten, wie es in den Experimenten von Eavey und Miller (1984) beobachtet wurde, und den Zivilisationsleistungen in anonymen Gesellschaften, wie der repräsentativen Regierungsform oder dem westeuropäischen Wohlfahrtsstaat, einen Zusammenhang gibt (Gintis u. a. 2003: 154).

\section{a) Wie funktionieren Laborexperimente?}

Was ist nun unter einem Laborexperiment zu verstehen? Die Labore der experimentellen Wirtschaftsforschung bestehen aus einer gewissen Anzahl von Computerarbeitsplätzen, die durch einen Sichtschutz voneinander abgetrennt sind. Probanden können dort, sofern dies erforderlich ist, in anonymisierter Form zwischen Entscheidungsalternativen wählen, die durch den Leiter des Experiments vorstrukturiert werden. Diese Alternativen werden am Bildschirm visualisiert und basieren auf spieltheoretischen Annahmen über menschliches Verhalten in einer Situation strategischer Interaktion mit anderen Akteuren. Folglich lassen sich dort innerhalb einer Gruppe von Probanden Verhandlungssituationen simulieren, menschliches Verhalten in Dilemmasituationen erforschen usw. (Palfrey 2006). Der Anreiz der Probanden (sie werden gemäß ihrer „Rolle“ auch als Proposer und Responder bezeichnet), an solchen Experimenten teilzunehmen und sich getreu ihrer tatsächlichen Präferenzen zu verhalten, besteht darin, dass für die Teilnahme an solchen Experimenten reale Geldbeträge ausgezahlt werden, manchmal sogar mehrere Monatseinkommen (Cameron 1999). Es hängt nun von dem individuellen und kollektiven Verhalten der Probanden ab, ob sie eine hohe oder niedrige Auszahlung erhalten. Dabei haben einige sehr einfache Spiele eine entscheidende Bedeutung für die Theoriebildung.

\section{b) Einfache Ultimatumspiele, Diktatorspiele und Public-Goods-Spiele}

Nicht in allen Spielen verhalten sich Akteure gleichermaßen kooperativ oder interessiert an dem Wohl des anderen Spielers. Für viele Ergebnisse von Laborexperimenten liefert das self-regarding preference model gute Voraussagen für die Entscheidungen der Akteure (Cox 2001). Wenn Proposer und Responder in einem Spiel mit mehreren Spielern um eine bestimmte Geldsumme mit anderen Spielern konkurrieren und dieser Wettbewerb einer Situation mit vollständigen Verträgen entspricht, dann ist eine sehr ungleiche Verteilung der Auszahlungen an die Spieler für die Beteiligten akzeptabel (Roth u. a. 1991). In solchen Zusammenhängen verhalten sich Menschen durchaus egoistisch. Etwas anders verhalten sich Personen in Wettbewerbssituationen mit unvollständigen Verträgen, wie zum Beispiel in Arbeitsverträgen, die ein Grundgehalt gewähren, das nicht an eine konkret vereinbarte Leistung gebunden ist. In solchen Vertragssituationen hat der Arbeitnehmer (im Experiment der Responder) die Möglichkeit, seine Leistung zu variieren, indem er für ein festes Gehalt mehr oder weniger Leistungsbereitschaft zeigt. Wie sich in der Praxis und im Experiment herausstellt, gibt es einen ,positiven Zusammenhang zwischen gezahltem Lohn und erbrachter Leistung". ${ }^{1}$ Dies zeigt einerseits, dass ein faires Verhalten (angemessene Lohnzahlung) erwartet wird, und

1 Fehr/Gächter 1996: 32; Liebig/Schupp 2007; Fehr/Gächter 2000a. 
andererseits, dass Personen ihren Lohn im Verhältnis zur Arbeitsleistung nicht einfach maximieren, indem sie ein hohes Gehalt für eine geringe Leistung einstreichen. Fairnessverhalten dient somit nicht nur als ein Mittel zur Befriedigung rein materieller Bedürfnisse.

Dies demonstrieren vor allem die Ergebnisse des einfachen Ultimatumspiels sehr eindrücklich (Güth/Schmittberger/Schwarze 1982). Dieses Spiel bietet den Vorteil, dass die Probanden aufgrund des geringen Komplexitätsgrades keine besonderen strategischen Fähigkeiten besitzen müssen. Dadurch kann verlässlich geprüft werden, ob sie ein optimales Verhandlungsziel anstreben oder nicht. Das Spiel selbst besteht darin, eine fixe Geldsumme $S$ unter den Spielern aufzuteilen. Dabei kann der Proposer einen Teilbetrag $x$ anbieten, den der Responder annehmen oder ablehnen kann. Nimmt der Responder den Betrag an, dann erhält der Proposer $S-x$ und der Responder $x$. Lehnt der Responder den Betrag jedoch ab, dann erhalten beide Spieler nichts. Dieses Spiel bildet beispielsweise die Logik einer zeitgebundenen Rabattaktion ab.

\section{Schaubild 1}

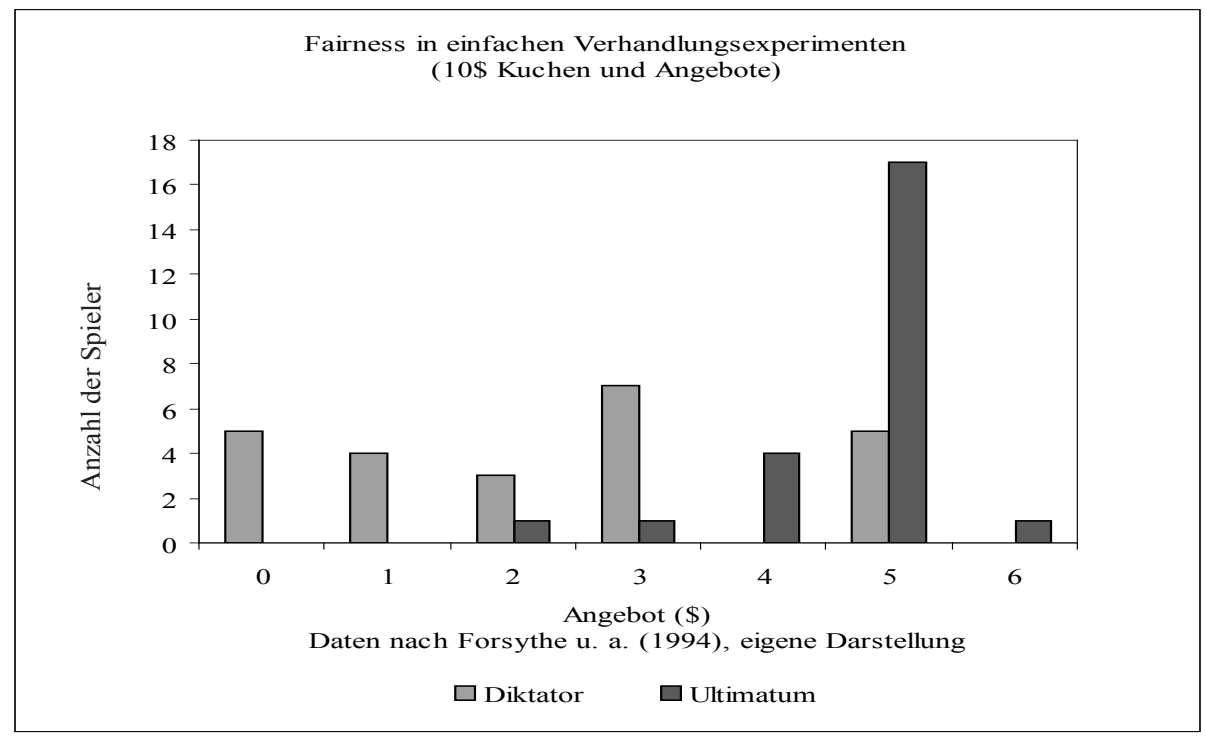

Im Diktatorspiel existiert hingegen keine Vetooption für den zweiten Spieler. Der Proposer diktiert eine Teilung der Geldsumme $(S-x, x)$. Dieses Spiel demonstriert zum Beispiel das Spendenverhalten von Lotteriegewinnern. Was geschieht nun in Experimenten, wenn diese Spiele im Labor gespielt werden? Die orthodoxe Wirtschaftswissenschaft würde für das Ultimatumspiel voraussagen, dass $x$ so klein wie möglich sein wird, weil der Proposer seine 
Auszahlung maximiert, wobei er voraussetzt, dass jedes $x>0$ vom Responder angenommen wird. Indes verhält sich die Mehrzahl der Probanden anders.

Schaubild 1 zeigt „mit typischen Daten“, dass die meisten Proposer im Ultimatumspiel $50 \%$ der Geldsumme anbieten. Eine ganze Reihe von Experimenten mit einfachen Ultimatumspielen zeigt ebenfalls, dass Responder in $50 \%$ der Fälle ein Angebot $x<20 \%$ ablehnen. Camerer und Fehr (2004) interpretieren dieses Verhalten als negative Reziprozität, was bedeutet, dass die Responder auf ein von ihnen als unfair wahrgenommenes Angebot bestrafend reagieren. Die meisten Proposer bieten, vermutlich die Bestrafung antizipierend, $50 \%$ von $S$.

Während die Motivation des Proposers auf den ersten Blick zweideutig bleibt, weil er durchaus kalkulieren mag, dass ein unfairer Betrag abgelehnt wird, ebenso wie er aus Freundlichkeit (Altruismus) heraus die verfügbare Summe in gleiche Hälften teilen könnte, ist das Verhalten des Responders zumindest in einem Punkt eindeutig: Er verzichtet auf Geld, wenn ihm diese Summe relativ zu der Summe des anderen Spielers zu gering erscheint. Er bewertet seinen immateriellen Nutzen, den er aus der Bestrafung seines unfair handelnden Spielpartners erhält, höher als den materiellen Nutzen einer relativ geringen Auszahlung. Diese Schlussfolgerungen ergeben sich freilich aus den Aggregatdaten. Einzelne Akteure mögen anders handeln, was die Vorhersagekraft des Modells aber nicht beeinträchtigt.

Die Ablehnung kleinerer Summen im Ultimatumspiel verletzt also die Bedingung rationaler Nutzenmaximierung. Zwar könnte nun angenommen werden, dass sich die Spieler durch „evolutionäres Training“ wie in einer iterierten Situation verhalten und daher bestrafen, um zukünftige Angebote zu erhöhen, jedoch haben Experimente gezeigt, dass dies nicht der Fall ist. Die Spieler sind sich der Einmaligkeit ihrer Begegnungen bewusst.

Nun ist die Frage: Handeln die Spieler nur fair, wenn sie mit Bestrafung rechnen müssen, oder handeln sie altruistisch und somit unabhängig von möglichen Vetopositionen des anderen Spielers? Diese Frage lässt sich teilweise beantworten, indem die Ergebnisse des Diktatorspiels betrachtet werden, da dort keine solche Vetoposition existiert. Schaubild 1 illustriert, dass die Anzahl der Spieler mit hohen Geboten drastisch sinkt, wohingegen einige Probanden gar nichts abgeben, andere lediglich $10 \%$. Interessanterweise geben allerdings auch im Diktatorspiel noch einige Probanden $50 \%$ des erhaltenen Geldbetrages ab, jedoch ist klar, dass die Vetoposition des zweiten Spielers mit entscheidend für das faire Verhalten der meisten Proposer im Ultimatumspiel ist (Bolton/Ockenfels 2000). Dies zeigt sich auch in den Ergebnissen so genannter Public-Goods-Spiele, bei denen sich herausstellt, dass die Mehrzahl der Probanden Trittbrett fährt, es sei denn, das Experiment lässt eine Bestrafung der Individuen zu, die sich nicht kooperativ verhalten.

Public-Goods-Spiele sind Spiele, in denen Dilemmasituationen simuliert werden, wie sie beispielsweise aus der Problematik der Verwaltung von natürlichen Ressourcen bekannt sind (Ostrom 1990). Sie werden folgendermaßen durchgeführt: Mehrere Spieler $n$ erhalten einen Betrag $y$, den sie ganz oder teilweise in einen gemeinsamen Topf einzahlen können $\left(g_{i}\right)$. Nachdem dies geschehen ist, erhält jeder Spieler außer der verbliebenen Summe von $y$ für jeden eingezahlten Euro eine Auszahlung $m<1$ aus dem gemeinsamen Topf, sodass sich der payoff jedes Spielers als: $\pi=y-g_{i}+m G$ darstellen lässt, wobei $G$ die Summe aller Einzahlungen darstellt und $m \leq 1 \leq m n$ sowie für $g_{i}\left(0 \leq g_{i} \leq y\right)$. Da $m G$ auch an die Spieler ausge- 
zahlt wird, die keinen Beitrag leisten, ist es in diesem Spiel individuell rational, Trittbrett zu fahren. Würde indes jeder Spieler $g_{i}=y$ einzahlen, dann ließe sich der Betrag im gemeinsamen Topf erhöhen und $m G$ für jeden Spieler maximieren, sodass auch der payoff jedes Spielers anstiege (Camerer/Fehr 2004).

Anhand der Daten von Fehr und Gächter (2000b) lässt sich nun zeigen, dass die Spieler, auch wenn sie mehrmals hintereinander spielen, kooperationswillig bleiben, sofern die Möglichkeit der Bestrafung existiert. Wird diese Möglichkeit indes genommen, bricht die Kooperation nach ein paar Spielrunden zusammen und zwar unabhängig davon, ob dieses Spiel im partner, im stranger, oder im perfect stranger treatment gespielt wird, also unabhängig davon, ob die Teilnehmer, weil sie im Experiment mehrfach miteinander interagieren, eine Reputation für kooperatives Verhalten aufbauen können oder nicht.

Wie im Gefangenendilemma sorgt hier jeder Spieler für einen suboptimalen payoff, indem er aufhört, einen Beitrag zu den „Gemeinschaftsgütern“ zu leisten. Dass die Möglichkeit der Bestrafung einen solch positiven Effekt hat, ist insofern bemerkenswert, als bestrafendes Verhalten ebenfalls mit Kosten verbunden ist. Die klassische Wirtschaftstheorie würde daher voraussagen, dass sich die Dilemmasituation für die Spieler durch die Möglichkeit der Bestrafung nicht verbessert.

\section{Schaubild 2}

Durchschnittliche Beiträge über Zeit (Public-Goods-Spiel) im Partner-, Stranger- und Perfect Stranger treatment (zuerst mit Bestrafung)

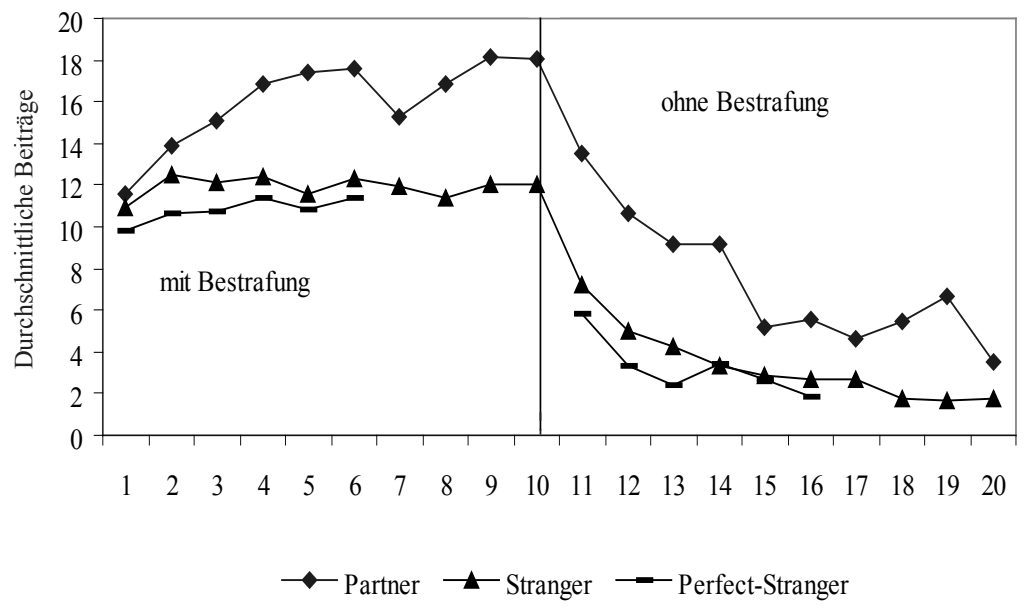

Daten: Fehr/Gächter (2000b), eigene Darstellung 
Um zu erklären, weshalb manche Probanden die Kosten der Bestrafung auf sich nehmen, argumentiert die Forschung uneinheitlich mit den Begriffen Reziprozität und Altruismus. Diese Uneinheitlichkeit ist dadurch entstanden, dass einige Forscher davon ausgehen, eine spezifische Form der Reziprozität könne altruistisch genannt werden (Gintis 2003), andere hingegen der Auffassung sind, dieses Verhalten sei immer an strategische Reziprozität gebunden und daher zwar kostenträchtig, aber in gewissem Maße selbstbezogen.

So sind sich die Vertreter der neueren Altruismusthese mit den Vertretern strategischer Reziprozitätstheorien darin einig, dass menschliches Kooperationsverhalten nicht als unbedingtes Verhalten gedeutet werden kann. Vielmehr beachten Akteure sehr genau, in welchen Umwelten sie agieren, und passen ihr Verhalten dementsprechend an. Die eigentliche Herausforderung der Forschung besteht folglich darin, diese Bedingungen besser kennenzulernen. Genau dieses Interesse steht auch im Zentrum der zeitgenössischen Debatte über altruistisches Verhalten.

\section{c) Altruismus in den neueren spieltheoretischen Ansätzen}

Trivers veröffentlichte bereits 1971 einen Artikel, in dem er ein Modell vorschlägt, das altruistisches Verhalten erklären soll. Warum, so fragt er, springen Menschen in einen See, um dort einen Ertrinkenden zu retten, obwohl sie dadurch selbst in Gefahr geraten? Er geht davon aus, dass es sich hierbei um reziprok altruistisches Verhalten handelt. Unter bestimmten Bedingungen lohnt es sich für Individuen, sich derart zu verhalten, weil sie hierdurch ihrerseits eine Bereitschaft in der Gruppe erzeugen, im Falle einer Bedrohung für das eigene Leben Hilfe anzubieten. Dieses Modell ist jedoch nur unter bestimmten Bedingungen gültig: ,(1) when there are many such altruistic situations in the lifetime of the altruists, (2) when a given altruist repeatedly interacts with the same small set of individuals, and (3) when pairs of altruists are exposed, symmetrically“ to altruistic situations" (Trivers 1971: 37). Diese Bedingungen sind zum Beispiel in andauernden Kampfhandlungen während eines Krieges gegeben. Sie verlangen also nicht die Verwandtschaft der Gruppenmitglieder, sind aber den Lebensbedingungen von Verwandten nicht unähnlich. Das Modell macht ähnliche Annahmen, wie sie für die ,tit-for-tat"-Strategie in iterierten Spielen von Rapoport formuliert wurden (Axelrod 2005: 28 f.).

Im Gefangenendilemma wäre nach Trivers ein Kooperationsergebnis „A kooperiert und B kooperiert“ eine altruistische Handlung der beiden Spieler, denn sie versuchen nicht, ihren individuellen Nutzen (Freiheit) zu maximieren, sondern nehmen Kosten auf sich, um eine geringe Strafe zu erhalten und somit den sucker's payoff sowie die beidseitige Bestrafung durch wechselseitigen Verrat zu vermeiden. Daran lässt sich aber erkennen, dass wir es hier nicht mit „unconditional kindness“ (Fehr/Gächter 2000a) zu tun haben, sondern dass erst die oben beschriebenen Bedingungen erfüllt sein müssen (insbesondere die Wiederholung des Spiels), damit sich Individuen reziprok altruistisch verhalten. Bowles und Gintis (2006) kritisieren daher zu Recht, dass Trivers Altruismuskonzept auf mutualism beruhe und nicht zur Erklärung von Kooperationsformen in anonymen Gesellschaften tauge, wie zum Beispiel einer umverteilenden Sozialpolitik. Es erklärt auch nicht die Spielergebnisse von 
one-shot games wie dem einfachen Ultimatumspiel, in denen keine Wiederholung des Spiels vorgesehen ist.

Um Spielergebnisse in nicht iterierten Spielen erklären zu können, die unter Bedingungen von Anonymität entstehen, sind meines Erachtens vor allem drei wichtige Theorien entwickelt worden: (1) Theorien der Ungleichheitsaversion, (2) Theorien reziproker Freundlichkeit und (3) Theorien starker Reziprozität.

Während Theorien der Ungleichheitsaversion auf die Altruismusannahme verzichten, setzt die Theorie starker Reziprozität altruistisches Verhalten voraus und erklärt seine Existenz mithilfe eines evolutionär spieltheoretischen Ansatzes. Die Theorie reziproker Freundlichkeit ist ebenfalls nicht leicht mit Altruismusannahmen vereinbar - ihr Hauptaugenmerk liegt auf der strategischen Reziprozität von Akteuren, die „Gleiches mit Gleichem“ belohnen.

Ungleichheitsaversion beruht im Kern auf der Annahme, dass die Spieler bei ihren Entscheidungen einerseits ihren absoluten Nutzen berücksichtigen, andererseits jedoch auch den relativen Nutzen in Bezug zum gesamten payout in ihre Kalkulation einbeziehen. Fällt der relative Nutzen für einen Spieler zu gering aus, dann zieht er es unter Umständen vor, den anderen Spieler zu bestrafen, anstatt selbst eine relativ geringe Auszahlung einzustreichen. In diesem Modell sind Spieler also bestrebt, Ungleichheit zu reduzieren. Bestrafendes Verhalten, wie zum Beispiel im Ultimatumspiel, eignet sich hierfür in ausgezeichneter Weise, weil diese Entscheidung beide Spieler mit einer gleichen Auszahlung enden lässt: null.

Die prominentesten Modelle der Ungleichheitsaversion stammen von Bolton/Ockenfels (2000) und Fehr/Schmidt (1999). Die eigentliche Innovation des Modells von Bolton und Ockenfels besteht darin, dass es auf einfache Weise ganz unterschiedliche Verhaltensweisen erklären kann: Rein selbstsüchtiges Verhalten unter Marktbedingungen lässt sich damit ebenso abbilden wie z. B. reziprokes Verhalten von Arbeitnehmern gegenüber Arbeitgebern.

Somit handelt es sich aber nicht um eine allgemeine Theorie der Verteilungsgerechtigkeit. Das Interesse eines Spielers, Ungleichheit zu reduzieren, darf nicht als ein abstraktes Gemeinwohlinteresse missverstanden werden. Im Gegenteil, das Modell geht davon aus, dass die eigene Auszahlung einigermaßen fair sein muss, es setzt nicht voraus, dass die Auszahlung an Dritte diesen Fairnessprinzipien entspricht. Wie Güth und van Damme (1998) mit einem Ultimatumspiel für drei Spieler zeigen, ist dies in Experimenten auch nicht der Fall. Werden einem dritten Spieler, der keine Vetoposition besitzt, nur relativ geringe Auszahlungen überlassen, dann ist dies kein Grund für den Responder zu intervenieren. Er lehnt keine Auszahlung ab, die Spieler Drei in unfairer Weise benachteiligt. Daher erkennen die Vertreter der Theorie der Ungleichheitsaversion keine altruistischen Handlungsmotive in den Handlungsweisen der Spieler. Schließlich sorgen die aktiv beteiligten Probanden nur untereinander für eine faire Auszahlung.

Gegen diese Schlussfolgerung bringen die Vertreter der Theorie reziproker Freundlichkeit (Falk/Fischbacher 2006) den Einwand vor, dass wir über die Motivation der ungleichheitsaversen Spieler nur erfahren, dass sie tendenziell gleiche payoffs gegenüber tendenziell ungleichen vorziehen. Das Modell betrachtet also nur die Konsequenzen einer Handlung, nicht jedoch die Intentionen des Spielers.

In Theorien reziproker Freundlichkeit wird indes davon ausgegangen, dass die Intentionen eines Akteurs die Kooperationsbereitschaft des anderen Akteurs entscheidend beeinflussen 
(Rabin 1993). Diese Annahme wird in der Tat durch die Ergebnisse einiger Experimente, wie sie Falk, Fehr und Fischbacher (2003) mit dem Ultimatumspiel durchgeführt haben, bestätigt. Ein bestimmtes Angebot $x$ wird darin häufiger zurückgewiesen, wenn der Responder die Intention unfairen Verhaltens seitens des Proposers anhand dessen Wahlmöglichkeiten nachvollziehen kann. Schränkt der Forscher die Möglichkeiten des Proposers hinsichtlich des anzubietenden Betrages $x$ ein und wird der zweite Spieler über diese Wahlmöglichkeiten informiert, so bewertet der zweite Spieler den gleichen Betrag $x$, den er unter Umständen in einem Spiel ohne diese Informationen als unfair bewertet hat, jetzt als faireren Betrag. Entsprechend variieren die Zurückweisungsraten im Ultimatumspiel (Falk/Fischbacher 2006: 295). Der Kritik der Autoren zufolge gibt es also Daten, die mit reiner Ungleichheitsaversion nicht erklärt werden können: „In Bolton and Ockenfels (2000), and Fehr and Schmidt (1999), players reciprocate to reduce inequity [...] they assume that fairness intentions are irrelevant. Distributive consequences of an action alone trigger reciprocal actions. Second, players reward or sanction only if this reduces inequity. This differs from our model, where people reciprocate perceived kindness or unkindness [...]“ (Falk/Fischbacher 2006: 310).

Bolton und Ockenfels (2005) haben diese beiden unterschiedlichen Ansätze in einer weiteren Studie einem stress test unterzogen. Darin entwickeln sie archetypische Formen für ein Verteilungsmodell (Ungleichheitsaversion) und ein Kindness-Modell (reziproke Freundlichkeit). Mithilfe dieser Modelle schätzen sie die Ergebnisse von Ultimatumspielen, um zu sehen, ob das Verteilungsmodell bzw. das Kindness-Modell unterlegen ist.

Es zeigt sich, dass die Kritik der Verfechter des Kindness-Modells nicht unberechtigt ist. Menschen reagieren reziprok auf Freundlichkeit und berücksichtigen bei ihren Sanktionen die Absichten anderer Akteure. Dies kann reine Ungleichheitsaversion deshalb nicht berücksichtigen, weil das Modell eine gleiche Verteilung (50/50) als einen festen Referenzpunkt für faires Verhalten modelliert. Wenn es einem Akteur nicht möglich ist, in dieser Weise zu teilen, und ein anderer Akteur darüber informiert ist, so mag er eine Aufteilung, welche dem 50/50Ideal am nächsten kommt, ebenfalls akzeptieren. Ungleichheitsaversion müsste in Hinsicht auf die möglichen Entscheidungsalternativen des ersten Spielers flexibler sein, um dies zu erklären.

Was bedeutet dies für die Altruismusthese? Menschen können durchaus großzügig sein, wenn sie wissen, dass ihre Mitmenschen unter eingeschränkten Bedingungen handeln. Wirtschaftliche Zumutungen in beschäftigungspolitisch schweren Zeiten haben durchaus eine Aussicht, von den Wählern akzeptiert zu werden. Entscheidend ist indes, ob diese Zumutungen fair verteilt werden. Liegen für unfaire Verteilungen keine notwendigen Bedingungen vor, schwindet hierfür die Unterstützung. Altruismus im Sinne unbedingter Freundlichkeit lässt sich folglich mit diesen Theorien und den oben erwähnten Daten nicht zeigen.

Es gibt jedoch noch einen dritten Ansatz, der die Altruismusthese nicht verwirft. Die Vertreter dieses Ansatzes bezeichnen den Wunsch der Menschen, unfaire Handlungsweisen auf eigene Kosten zu bestrafen oder auf eigene Kosten zu kooperieren, als altruistisches Verhalten. ${ }^{2}$ Aber auch sie gehen davon aus, dass sich ihre Probanden im Labor wie conditional cooperator oder conditional punisher verhalten (Fong/Bowles/Gintis 2005: 287). Sie nehmen 
folglich ebenfalls an, dass sich Menschen reziprok verhalten, allerdings nach einem Prinzip, das sie strong reciprocity nennen.

Dieses Prinzip unterscheidet sich von den oben erwähnten Reziprozitätsannahmen dadurch, dass Personen bereits kooperative Absichten verfolgen, bevor sie die konkrete Situation kennenlernen, in der sie interagieren müssen. Sie wissen folglich nicht, ob sie mit einem spiegelbildlichen Verhalten seitens der potenziellen Kooperationspartner rechnen dürfen oder nicht. Diese Prädisposition (Gintis 2003: 161) erhalten Akteure durch die Internalisierung von Normen.

Obwohl für diese Annahme dieselben experimentellen Daten zugrunde gelegt werden, wie sie oben beispielhaft dargestellt wurden, gelangen die Autoren zu einem anderen Schluss als die Verfechter der Ungleichheitsaversion. Dies liegt daran, dass sie den Referenzpunkt für faires Verhalten als kulturell normiert ansehen. Das 50/50-Maß mag ein akzeptabler Referenzpunkt für faires Teilen in westlichen Gesellschaften sein, längst jedoch nicht in allen Gesellschaften der Welt. Demzufolge müsste kooperatives bzw. bestrafendes Verhalten kulturell variieren, was für die vergleichende Analyse von Wohlfahrtsstaaten von erheblicher Bedeutung sein könnte. Wenn die Bereitschaft zur Unterstützung sozialpolitischer Programme in gewissen Grenzen kulturell normiert wäre, dann ließen sich die Unterschiede in der sozialpolitischen Performanz von Wohlfahrtsstaaten vielleicht aus individuellen Verhaltensmustern heraus erklären. Zudem wäre es denkbar, dass Wohlfahrtsstaaten über Zeit in Aufwärtsund Abwärtsspiralen geraten, die nicht nur etwas mit den gesamtwirtschaftlichen Rahmenbedingungen zu tun haben, sondern auch mit der Unterstützung oder der Verletzung von sozialen Normen.

Für die kulturelle Varianz kooperativen bzw. bestrafenden Verhaltens finden Henrich u. a. (2004a) tatsächlich beeindruckende empirische Belege in Experimenten mit fünfzehn Naturvölkern (small-scale societies) in Peru, Tansania, Bolivien, Ecuador, der Mongolei, Chile, Papua Neu Guinea, Zimbabwe, Kenia, Paraguay und Indonesien. Das durchschnittliche Proposergebot im Ultimatumspiel mit Studenten in westlichen Gesellschaften liegt zwischen $43 \%$ und $48 \%$. In dem oben beschriebenen Sample indes liegt es zwischen $26 \%$ und $58 \%$. Auch die Zurückweisungsraten zwischen diesen Gesellschaften variieren erheblich, wobei das Proposergebot in Experimenten mit manchen Gesellschaften nie zurückgewiesen wurde, in anderen erfolgten die Zurückweisungen ähnlich wie in westlichen Gesellschaften. In Experimenten mit wieder anderen Gesellschaften zeigte sich eine überdurchschnittlich hohe Zurückweisungsrate, sogar Angebote von über $50 \%$ wurden abgelehnt (Gintis u. a. 2003: 158).

Laut Henrich u. a. (2004a) ist dieses Verhalten stark durch die unterschiedlichen Lebensbedingungen geprägt, in denen sich die Mitglieder dieser Gesellschaften befinden. Zum Beispiel sind die Lamalera in Indonesien Walfänger, die für den Fang zusammenarbeiten müssen und ein sehr kompliziertes System kennen, ihren Fang unter den Clanmitgliedern aufzuteilen. Entsprechend großzügig waren ihre Angebote im Ultimatumspiel. Während der Modalwert von $50 \%$ dem westlichen Modalwert entspricht, gab es eine Reihe von Probanden, die hyperfaire Angebote zwischen $60 \%$ und $90 \%$ machten (Alvard 2004). Die Machiguenga im peruanischen Amazonas dagegen jagen und fischen in kleinen Familienverbünden. Für ihr Überleben sind sie auf keinerlei kooperatives Verhalten jenseits der eigenen Verwandtschaft 
angewiesen. Entsprechend niedrig lag der Modalwert der Machiguenga in Experimenten mit dem Ultimatumspiel (15\%), wobei die durchweg niedrigen Proposergebote $(x<25 \%) \mathrm{im}$ Experiment beinahe immer akzeptiert wurden (Henrich/Smith 2004). Je stärker eine Gesellschaft eine Marktintegration erfahren hatte, also anonyme Transaktionen kannte, desto kooperativer zeigte sie sich in der Studie von Henrich u. a. (2004b).

Die Nivellierung westlicher Lebensbedingungen und die starke Marktintegration in diesem Teil der Welt könnte somit erklären, weshalb Ergebnisse in Experimenten hier relativ ähnlich ausfallen. Dennoch weist die Studie von Henrich u. a. (2004a) darauf hin, dass kooperatives Verhalten auch hier normgebunden sein könnte und somit gewissen kulturellen Einflüssen unterliegt, welche die Prädisposition kooperativen Verhaltens prägen: „strong reciprocity is a virtually universal behavioural template, $[\ldots]$ its expression is strongly affected by the culture of the particular society in which it is expressed" (Gintis 2003: 169).

Handeln Menschen also normgebunden altruistisch, und zwar in manchen Kulturen stärker als in anderen? Ob diese Frage bejaht oder verneint wird, ist von folgender Annahme abhängig: Wird altruistisches Verhalten als ein Muster betrachtet, das mit Reziprozität vereinbar ist, wie dies die Verfechter des Ansatzes starker Reziprozität vorschlagen, dann lässt sich Verhalten, das in nicht iterierten Situationen auftaucht und Kosten für den betreffenden Akteur verursacht, auch als altruistisch bezeichnen. Altruismus ist jedoch auch dann nicht mit unconditional kindness identisch. Sie ist kulturell normierte Freundlichkeit zum Zwecke der Lösung von Dilemmasituationen.

Der Altruismusbegriff führt hier allerdings ein wenig in die Irre, weil er suggeriert, dass die Varianz kooperativen Verhaltens etwas mit einer Kultur des Gebens und individuell anerzogener Güte zu tun hat, die auf Umweltbedingungen nicht achtet. Die Experimente und die Untersuchungen von Henrich u. a. (2004a) weisen aber in eine ganz andere Richtung: Sie zeigen, dass es unterschiedliche regionale Reziprozitätsgleichgewichte gibt, dass also Proposer und Responder in trainierten Varianten auf strategische Handlungen ihres Gegenübers reagieren.

Der Altruismusbegriff ist folglich eher als Kampfbegriff in den Wirtschaftswissenschaften zu verstehen. Er schafft eine plakative Abgrenzung zur klassischen und neoklassischen Wirtschaftstheorie. Aus diesem Grund wird Altruismus auch leicht als eine äußerst extreme Gegenposition zum homo oeconomicus gedeutet. Liest man die Theorien der experimentellen Wirtschaftsforschung jedoch ohne diesen Vorbehalt, ergeben sich daraus eine Reihe von interessanten Hypothesen über die Entwicklung des modernen Sozialstaates.

\section{Verteilungskonflikte in westeuropäischen Sozialstaaten}

Wie kann nun die Policyanalyse von der experimentellen Forschung profitieren? Es gibt sicherlich viele denkbare Anknüpfungspunkte. Ich will hier jedoch nur einen vorschlagen, und zwar zur Vertiefung des Verständnisses von Konflikten in modernen Sozialstaaten. Diese Konflikte sehen in verschiedenen Ländern ganz unterschiedlich aus, aber es lässt sich dennoch eine Gemeinsamkeit entdecken. Sie besteht in der Verringerung der Absicherung gegen die kohortenspezifischen Lebensrisiken junger Menschen. Die meisten Sozialausgaben gehen OECD-weit in die Finanzierung von Alterssicherung und Gesundheit (OECD 2004). Auch wenn klar sein dürfte, dass diese Lastenteilung mit dem demografischen Wandel in Westeuropa 
zu tun hat, so gibt es doch davon unabhängige Probleme, die mit den oben vorgestellten Modellen gut erklärt werden können. Zum Beispiel ist nicht ohne Weiteres ersichtlich, warum die Kürzungen von Sozialleistungen im Bereich der Arbeitslosenunterstützung einen gewissen Zuspruch finden, während das Risiko zunimmt, von Arbeitslosigkeit betroffen zu sein (Fong/Bowles/Gintis 2005). Gerade die Vertreter starker Reziprozität, die die Altruismusannahme am stärksten unterstützen, machen auf diesen Zusammenhang aufmerksam. Wie lässt sich die Verweigerung von Wohlfahrt mit Altruismus erklären?

Zunächst muss man klarstellen, dass das Niveau der gesamten öffentlichen Sozialausgaben in 11 von 17 westeuropäischen Sozialstaaten (in den neunziger Jahren) nicht sinkt, sondern steigt. Die einzigen westeuropäischen Staaten, die im Jahr 2001 gegenüber dem Jahr 1990 eine etwas geringere öffentliche Sozialausgabenquote (gemessen in Prozent am BIP) aufzuweisen hatten, waren Schweden (-1 \%), Dänemark (-0,1\%), Norwegen (-0,8 \%), Luxemburg $(-1,1 \%)$, die Niederlande (-5,8\%) und Irland (-4,8 \%) (OECD 2004).

Im Falle der skandinavischen Länder fällt der Rückgang gegenüber 1990 äußerst gering aus. Ab Mitte der neunziger Jahre nahmen diese Länder, nachdem die Sozialausgabenquote von einem hohen Niveau aus extrem angestiegen war, unter anderem um die Folgeerscheinungen der Rezession von 1992/93 zu bekämpfen, eine erhebliche Reduzierung vor. Dies gilt besonders für Schweden. Der dort beobachtbare Rückgang der Sozialausgaben lässt sich jedoch als eine Korrektur eines äußerst großzügigen öffentlichen Ausgabengebarens während der neunziger Jahre verstehen, nicht als eine Reduzierung gegenüber vorangegangenen Dekaden: 2001 lagen die Sozialausgaben der skandinavischen Länder durchweg höher als zu Beginn der achtziger Jahre. Irland verzeichnet ebenfalls einen sehr speziellen Trend, weil es aufgrund niedriger Löhne und anderer Faktoren zu einem besonders attraktiven Investitionsraum für ausländische Unternehmen geworden ist. Typisch für die meisten Staaten ist also eine weiterhin hohe Finanzierungslast für sozialpolitische Maßnahmen oder sogar eine weitere Expansion des Wohlfahrtsstaates, nicht etwa eine Reduzierung seiner Ausgaben.

Die Krise des Sozialstaates entsteht bislang also nicht durch eine Reduzierung der Haushaltsmittel, sondern durch eine Explosion auf der Kostenseite. Überalterung einerseits und Arbeitslosigkeit andererseits nehmen die öffentlichen Versicherungssysteme von zwei Seiten her in die Zange. In dieser Situation entstehen verschiedene Konflikte, weil die Umverteilungsmasse relativ gesehen schrumpft. Wenn es zutrifft, dass Menschen altruistisch handelnkönnen, ist dann von diesemAltruismuspotenzial eine Lösung dieses Verteilungskonfliktes zu erwarten, indem bestimmte Gruppen ,,auf Geld verzichten“, um einer neuen Umverteilungspolitik auf die Beine zu helfen?

\section{Kostenexplosion und Verteilungsgerechtigkeit}

Insbesondere die Alterssicherung nimmt einen immer stärker anwachsenden Teil der Sozialausgaben in Anspruch. Somit kommen die verausgabten Mittel der europäischen Sozialhaushalte immer mehr bestimmten Alterskohorten zugute. Die Lebensrisiken junger Menschen werden vor allem in den liberalen und konservativen Wohlfahrtsstaaten (Esping-Andersen 1990 ) immer weniger abgesichert. Im europäischen Durchschnitt (EU-15) sind die Ausgaben für Alterssicherung zwischen 1990 und 2001 von 7,9 \% auf 8,8 \% am BIP gestiegen, während 
die Ausgaben für Arbeitslosigkeit im gleichen Zeitraum von 1,5\% auf 1,2 \% am BIP sanken (OECD 2004).

So erzwingt der Wohlfahrtsstaat neuerdings eine systematische Verletzung von Gerechtigkeitsnormen, denn wie Schaubild 3 zeigt, schrumpfen in vielen Staaten die Pro-Kopf-Ausgaben für Arbeitslosigkeit relativ zu den Pro-Kopf-Ausgaben für Alterssicherung. Hierdurch entsteht einerseits ein Verteilungskonflikt zwischen Alterskohorten, andererseits aber auch ein Verteilungskonflikt unter jungen Menschen, die nun immer mehr darauf achten, ob die immer geringeren Sozialausgaben in ihrer Generation leistungsgerecht verteilt werden. Zu den stratifizierenden Effekten des Wohlfahrtsstaates, wie sie Esping-Andersen (1990) beschreibt, treten nun also noch weitere sozialpolitisch verzerrende Wirkungen hinzu, welche die sozialen Folgen einer freien Wirtschaft nicht nur dämpfen, sondern auch in bestimmter Weise verschärfen. Die Politikwissenschaft ist gegenwärtig damit beschäftigt, dieses Phänomen zu untersuchen, indem sie die Effekte sozialstaatlicher Reformen in verschiedenen Wohlfahrtsstaatstypen, also rein auf der Makroebene, untersucht (Lütz/Czada 2004).

\section{Schaubild 3}

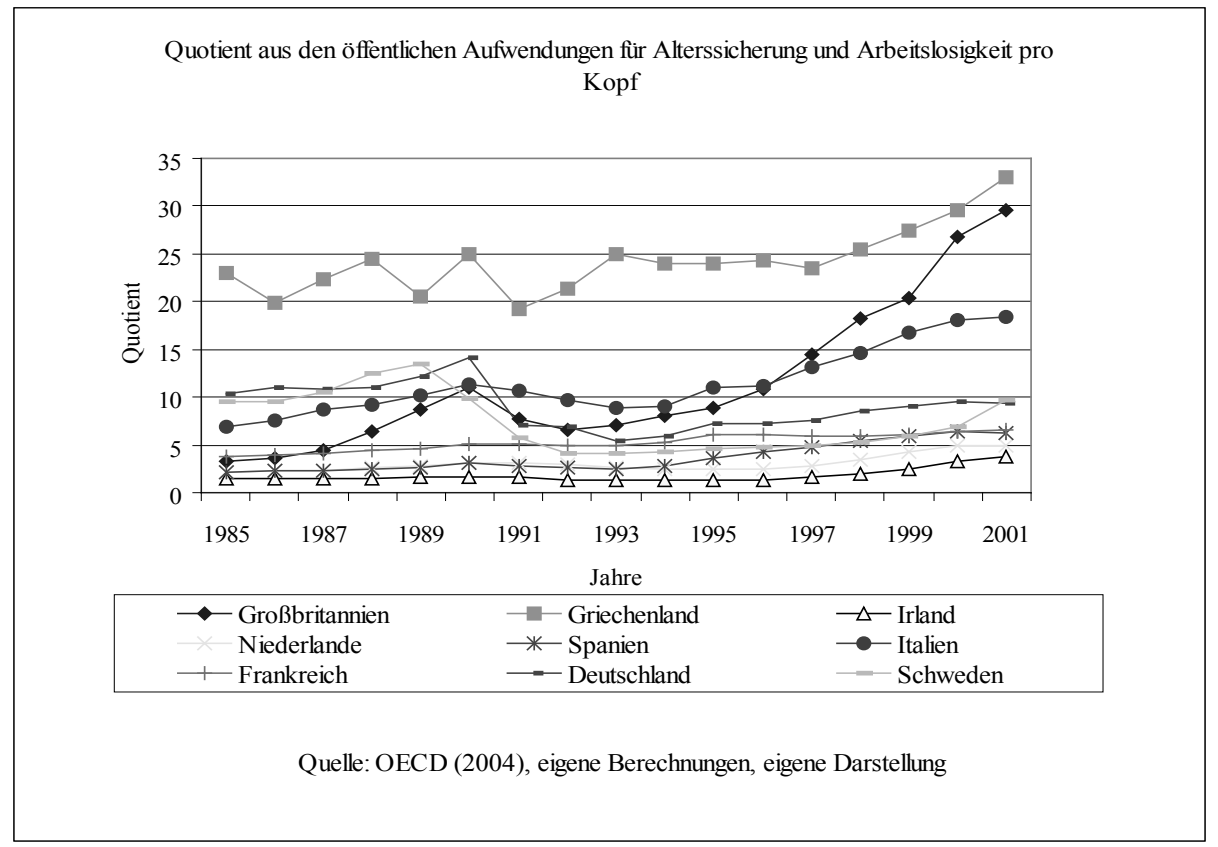

Im Unterschied dazu besteht der spezifische Beitrag der experimentellen Forschung jedoch darin, die Zusammenhänge zwischen individuellem Verhalten und politischen sowie wirt- 
schaftlichen Makrophänomenen zu erklären. In diese Richtung gehen auch einige Analysen der ökonomischen Soziologie (Mau 2004), in denen Wohlfahrtstaatlichkeit mithilfe einer Reziprozitätsannahme erklärt wird.

Nun stellt sich die Frage, ob das altruistische Handlungsmotiv das oben beschriebene Dilemma löst und für einen Verzicht derjenigen sorgt, die nicht von Arbeitslosigkeit betroffen sind. Aus der experimentellen Forschung ist bekannt, dass das altruistische Handlungsmotiv nicht zwangsläufig dazu führt, Umverteilungsprogramme bedingungslos zu unterstützen. In einer Aufwärtsspirale positiver Reziprozität kann es zu einer sehr ausgedehnten Unterstützung wohlfahrtsstaatlicher Maßnahmen kommen, jedoch kann Altruismus auch das Gegenteil bewirken. Besonders wenn die zur Verfügung stehende Umverteilungsmasse immer kleiner wird, stärkt das altruistische Handlungsmotiv den Wunsch danach, diejenigen zu bestrafen, welche von diesem System unverhältnismäßig stark profitieren.

Eine interessante Prüfung dieser These findet sich bei Fong/Bowles/Gintis (2005). Sie haben für den US-amerikanischen Fall und mithilfe von Umfragedaten des Gallup Social Audit Survey untersucht, ob Menschen wohlfahrtsstaatliche Programme im Sinne einer allgemeinen Solidarität für ihre Mitbürger unterstützen oder nicht. Dabei kam heraus, dass die Zustimmung zu staatlichen Unterstützungsleistungen vor allem von der Wahrnehmung der Arbeitsmoral der Empfängergruppen abhängig ist. Diese Variable ist entscheidender als die Einkommensstruktur der Haushalte. Diejenigen, die Arbeitslosigkeit oder Armut für ein erlittenes Schicksal halten, für das nicht in der Verantwortung der Person liegende Faktoren ursächlich sind, unterstützen solche Maßnahmen, während diejenigen, die der Auffassung sind, man könne diesen Lebensrisiken durch harte Arbeit und eine hohe Risikobereitschaft entkommen, sie eher ablehnen (Fong/Bowles/Gintis 2005: 288-291).

Das altruistische Handlungsmotiv, wie Gintis u. a. (2003) es verstehen, ist somit an die Wahrnehmung fairer Auszahlungen gekoppelt; es gründet geradezu auf dem Prinzip der Leistungsgerechtigkeit, denn es muss, wie oben gezeigt, eben auch als ein reziprokes Handlungsmuster verstanden werden. Ein Anspruch auf Wohlfahrt muss in den Augen vieler durch vorangegangene Arbeit oder ernstes Bemühen darum erworben werden. Wohlfahrt ohne diese Anstrengung nehmen viele Menschen als eine ungerechte Auszahlung wahr. Je stärker nun die Verteilungsgerechtigkeit sinkt, desto mehr thematisiert die Öffentlichkeit und die Politik die Verdienstwürdigkeit von Leistungsempfängern, weil die schrumpfende Verteilungsmasse die Bestrafung von Trittbrettfahrern immer notwendiger erscheinen lässt. Altruismus kann so zur Abschaffung sozialer Bürgerrechte, wie sie Marshall (1992) vor Augen hatte, führen, weil die Idee einer unbedingten Anspruchsberechtigung diesem Verhaltensmuster widerspricht. Dieser Zusammenhang zeigt, wie missverständlich der Altruismusbegriff ist.

Die oben erwähnten Umfragedaten spiegeln jedoch die Ansichten amerikanischer Befragter wider; sie liefern also ein Bild, wie es für liberale Wohlfahrtsstaaten typisch sein dürfte. Es stellt sich die Frage, ob diese Einstellungsmuster länderspezifisch variieren, welche Ursache dies hat und ob bestimmte Verteilungskonflikte durch „faire Zumutungen“ eher gelöst werden können als in anderen Ländern. Es wäre interessant zu sehen, welche individuellen Verhaltensmuster in Fällen (Länder, Regionen etc.) erkennbar sind, in denen dieser Konflikt stärker eskaliert bzw. in denen ein etwas ausgeglicheneres Lastenverhältnis zwischen den Generationen 
besteht, wie in einigen skandinavischen Ländern. Die experimentelle Forschung kann zu diesen Fragen interessante Theorien und Hypothesen liefern. Vor allem vermittelt sie ein klares Bild davon, dass die Bereitschaft zu teilen an ganz spezifische Bedingungen geknüpft ist und daher nicht zu erwarten ist, dass ein brach liegendes Altruismuspotenzial, an das in politischen Reden gerne appelliert wird, dazu beiträgt, dieses Problem zu entschärfen.

\section{Fazit}

Der Altruismusbegriff ist in der wissenschaftlichen und in der öffentlichen Debatte zu neuer Popularität gelangt. Inzwischen wird er in vielen Disziplinen unterschiedlich gefasst. Eine spezifische Begriffstradition ist jedoch in der experimentellen Forschung, neuerdings der experimentellen Wirtschaftsforschung, erkennbar. Der Beitrag hat diese Tradition aufgehellt und zugleich erläutert, dass die prominenten Vertreter der Altruismusthese hierunter keineswegs eine umweltunabhängige Freundlichkeit verstehen, sondern ebenfalls bestimmte Bedingungen an dieses Verhaltensmuster knüpfen. Die drei prominentesten Ansätze in der experimentellen Wirtschaftsforschung, Ungleichheitsaversion, reziproke Freundlichkeit sowie starke Reziprozität, nehmen zwar unterschiedliche Positionen gegenüber der Altruismusannahme ein, aber sie alle sehen Akteurshandeln entscheidend von strategischen Verhaltensmustern beeinflusst und unterscheiden sich daher eher in der Weise, ob und wie sie die Intentionen und Handlungsmotive von Akteuren in ihre Modelle einbauen. Ein besseres Verständnis dieser Zusammenhänge ist auch für die Politikwissenschaft von entscheidender Bedeutung, denn zwischen individuellen Verhaltensmustern und politikwissenschaftlich relevanten Makrophänomenen besteht eine wichtige Verbindung.

Die Theorien der experimentellen Wirtschaftsforschung sind auch für die Politikwissenschaft nützlich, weil sie sich seit geraumer Zeit mit Fairnessproblemen in Verteilungskonflikten befassen und gleichzeitig Hypothesen zu individuellem Verhalten in diesen Konflikten liefern, die in einem experimentellen Forschungsdesign verlässlich getestet werden können. Zwar verfügt auch die nicht experimentell geprägte Sozialwissenschaft über Möglichkeiten, z. B. durch Umfragen, Daten zu individuellen Einstellungsmustern zu erheben, aber gerade in diesem Bereich zeigen neuere Analysen, wie nützlich die Konzeptbildung anhand experimentell untermauerter Theorien sein kann. So folgen z. B. Liebig und Schupp (2007) der Theorie starker Reziprozität, um die Gerechtigkeitsorientierungen der Deutschen gegenüber ihren Erwerbseinkommen zu erklären.

Fairnessprobleme bilden im Zeitverlauf gesehen ein immer gravierender werdendes Problem in modernen Sozialstaaten. Seit dem Beginn der neunziger Jahre hat die materielle Ungleichheit in westlichen Demokratien fast in allen Staaten zugenommen (Förster/Mira D'Ercole 2005). Gleichzeitig steigen die allgemeinen Kosten für eine soziale Grundversorgung und so verringert sich, relativ betrachtet, die Verteilungsmasse, die dem Sozialstaat erlaubt, diese Ungleichheit zu bekämpfen. Hätten in dieser Situation alle Bürger gleiche Einschränkungen hinzunehmen, dann wäre zwar immer noch ein sozialpolitisches Problem gegeben, aber nicht in gleicher Weise ein Fairnessproblem entstanden. Wie sich jedoch zeigt, produzieren auch westliche Demokratien immer mehr spezifische Verlierergruppen. Dies zeigt sich im Bildungsbereich ebenso wie bei der gesellschaftlichen Integration von Minder- 
heiten, aber auch bei der Absicherung gegen Arbeitslosigkeit usw. Diese ungleiche Lastenverteilung birgt Probleme, die auf der Makroebene inzwischen gut beschreibbar sind und deren Ursache vielfach historisch erklärt werden kann (Esping-Andersen 1990, 1999).

Was aber geschieht auf der individuellen Ebene? Reagieren Individuen unterschiedlich auf diese Veränderungen? Und wenn sie unterschiedlich reagieren, liegen die Ursachen dafür tatsächlich in der verschiedenen Ausprägung sozialer Normen, wie Henrich u. a. (2004a) behaupten? Wenn dies zutrifft, geht dann die stärkere oder weniger starke Verletzung von Fairnessnormen auf diese kulturelle Prädisposition der Akteure zurück? Wenn auch dies zuträfe, dann lassen sich bestimmte Makrophänomene, wie die Varianz in der Unterstützung von Umverteilungsprogrammen, und somit die Entschärfung dieses Fairnessproblems aus individuellen Verhaltensmustern heraus erklären. Diese Fragen sind bisher ungeklärt und bieten der Politikwissenschaft viele Möglichkeiten, mithilfe einer Kombination verschiedener Methoden zu praxisrelevanten Antworten zu gelangen.

\section{Literatur}

Alvard, Michael S., 2004: The Ultimatum Game, Fairness, and Cooperation among Big Game Hunters, in: Henrich u. a. 2004a, 413-435.

Axelrod, Robert, 2005: Die Evolution der Kooperation, München (6. Aufl.).

Bolton, Gary/Ockenfels, Axel, 2000: ERC: A Theory of Equity, Reciprocity, and Competition, in: American Economic Review 90 (1), 166-193.

Bolton, Gary/Ockenfels, Axel, 2005: A stress test of fairness measures in models of social utility, in: Economic Theory 25 (4), 957-982.

Bowles, Samuel/Gintis, Herbert, 2006: The Evolutionary Basis of Collective Action, in: Weingast/Wittman 2006, 951-967.

Camerer, Colin/Fehr, Ernst, 2004: Measuring Social Norms and Preferences. Using Experimental Games: A Guide for Social Scientists, in: Henrich u. a. 2004a, 55-95.

Cameron, Lisa A., 1999: Raising the Stakes in the Ultimatum Game: Experimental Evidence from Indonesia, in: Economic Inquiry 37 (1), 47-59.

Cox, James C., 2001: On the Economics of Reciprocity (unveröffentlichtes Manuskript).

Eavey, Cheryl L./Miller, Gary J., 1984: Fairness in Majority Rule Games with a Core, in: American Journal of Political Science 28 (3), 595-609.

Esping-Andersen, Gøsta, 1990: The Three Worlds of Welfare Capitalism, Princeton/NJ. Esping-Andersen, Gøsta, 1999: Social Foundations of Postindustrial Economies, Oxford. Falk, Armin, 2004: Charitable Giving as a Gift Exchange: Evidence from a Field Experiment, London (CEPR Discussion Paper No. 4189).

Falk, Armin/Fehr, Ernst/Fischbacher, Urs, 2003: On the Nature of Fair Behavior, in: Economic Inquiry 41 (1), 20-26.

Falk, Armin/Fischbacher, Urs, 2006: A theory of reciprocity, in: Games and Economic

Behavior 54 (2), 293-315.

Fehr, Ernst/Fischbacher, Urs, 2003: The nature of human altruism, in: Nature 425, 785-791. 
Fehr, Ernst/Gächter, Simon, 1996: Soziale Kräfte und Lohnbildung. Einsichten aus der experimentellen Arbeitsmarktforschung, in: de Gijsel u. a. 1996, 11-51.

Fehr, Ernst/Gächter, Simon, 2000a: Fairness and Retaliation: The Economics of Reciprocity, in: Journal of Economic Perspectives 14, 159-181.

Fehr, Ernst/Gächter, Simon, 2000b: Cooperation and Punishment in Public Goods Experiments, in: The American Economic Review 90 (4), 980-994.

Fehr, Ernst/Schmidt, Klaus M., 1999: A Theory of Fairness, Competition, and Cooperation, in: The Quarterly Journal of Economics 114 (3), 817-868.

Fiorina, Morris P./Plott, Charles R., 1978: Committee Decisions under Majority Rule: An Experimental Study, in: The American Political Science Review 72 (2), 575-598.

Förster, Michael/Mira D'Ercole, Marco, 2005: Income distribution and poverty in OECD countries in the second half of the 1990s, Paris (OECD Social, Employment and Migration Working Paper).

Forsythe, Robert u. a., 1994: Fairness in Simple Bargaining Experiments, in: Games and Economic Behavior 6 (3), 347-369.

Fong, Christina M./Bowles, Samuel/Gintis, Herbert, 2005: Behavioural Motives for Income Redistribution, in: The Australian Economic Review 38 (3), 285-297.

Frey, Bruno S./Bohnet, Iris, 1996: Experiments, Theory - and Reality?, in: de Gijsel u. a. 1996, 340-359.

Gijsel, Peter de u. a. (Hrsg.), 1996: Ökonomie und Gesellschaft, Jahrbuch 13, Frankfurt a. M./New York.

Gintis, Herbert, 2000: Strong Reciprocity and Human Sociality, in: Journal of Theoretical Biology 206 (2), 169-179.

Gintis, Herbert, 2003: Solving the puzzle of prosociality, in: Rationality and Society 15 (2), 155-187.

Gintis, Herbert u. a., 2003: Explaining altruistic behavior in humans, in: Evolution and Human Behavior 24, 153-172.

Green, Donald P./Gerber, Alan S., 2003: The Underprovision of Experiments in Political Science, in: The ANNALS of the American Academy of Political and Social Science (ANNALS, AAPSS) 589 (1), 94-112.

Güth, Werner/Schmittberger, Rolf/Schwarze, Bernd, 1982: An Experimental Analysis of Ultimatum Bargaining, in: Journal of Economic Behavior and Organization 3 (4), 367388.

Güth, Werner/van Damme, Eric, 1998: Information, Strategic Behavior and Fairness in Ultimatum Bargaining: An Experimental Study, in: Journal of Mathematical Psychology 42 (2), 227-247.

Heckmann, James/Smith, Jeffrey, 1996: Social Experiments: Theory and Evidence, in: de Gijsel u. a. 1996, 186-213.

Henrich, Joseph u. a., 2001: In Search of Homo Economicus: Behavioral Experiments in 15 Small-Scale Societies, in: Economics and Social Behavior 91 (2), 73-78.

Henrich, Joseph u. a. (Hrsg.), 2004a: Foundations of Human Sociality. Economic Experiments and Ethnographic Evidence from Fifteen Small-Scale Societies. Oxford.

Henrich, Joseph u. a., 2004b: Overview and Synthesis, in: dies. 2004a, 8-54. 
Henrich, Joseph/Smith, Natalie, 2004: Comparative Experimental Evidence from Machiguenga, Mapuche, Huinca, and American Populations, in: Henrich u. a. 2004a, 125-167.

Liebig, Stefan/Schupp, Jürgen, 2007: Gerechtigkeitsprobleme im Wohlfahrtsstaat: Besteuerung, wohlfahrtsstaatliche Transfers und die Gerechtigkeit des eigenen Erwerbseinkommens, Berlin (DIW Berlin, Discussion Paper 690).

Lütz, Susanne/Czada, Roland (Hrsg.), 2004: Wohlfahrtsstaat - Transformation und Perspektiven, Wiesbaden.

Marshall, Thomas H., 1992: Bürgerrechte und soziale Klassen. Zur Soziologie des Wohlfahrtsstaates, Frankfurt a. M./New York.

Mau, Steffen, 2004: Welfare Regimes and the Norms of Social Exchange, in: Current Sociology $52(1), 53-74$.

$O E C D, 2004:$ Social expenditure database. Total public social expenditure (1980-2001) by main category, http://www.oecd.org.

Ostrom, Elinor, 1990: Governing the Commons. The Evolution of Institutions for Collective Action, Cambridge.

Palfrey, Thomas R., 2006: Laboratory Experiments, in: Weingast/Wittman 2006, 915-936.

Rabin, Mathew, 1993: Incorporating Fairness into Game Theory and Economics, in: The American Economic Review 83 (5), 1281-1302.

Roth, Alvin E. u. a., 1991: Bargaining and Market Behavior in Jerusalem, Ljubljana, Pittsburgh, and Tokyo: An Experimental Study, in: The American Economic Review 81 (5), 1068-1095.

Schram, Arthur, 2005: Artificiality: The tension between internal and external validity in economic experiments, in: Journal of Economic Methodology 12 (2), 225-237.

Trivers, Robert L., 1971: The Evolution of Reciprocal Altruism, in: The Quarterly Review of Biology 46 (1), 35-57.

Weingast, Barry/Wittman, Donald A. (Hrsg.), 2006: The Oxford Handbook of Political Economy, Oxford.

Korrespondenzanschrift:

Dr. Ulrich Glassmann

Universität zu Köln

Fakultät für Wirtschafts- und Sozialwissenschaften

Lehrstuhl für Vergleichende Politikwissenschaft

Gottfried-Keller-Straße 6

50931 Köln

E-Mail: ulrich.glassmann@uni-koeln.de

Web: http://www.politik.uni-koeln.de/kaiser/mitarbeiter/glassmann.htm

Der Autor ist wissenschaftlicher Mitarbeiter am Lehrstuhl für Vergleichende Politikwissenschaft der Universität zu Köln. 